\title{
WSAA 9
}

\section{H. Toruńczyk}

On infinite-dimensional manifolds

In: Zdeněk Frolík (ed.): Abstracta. 9th Winter School on Abstract Analysis. Czechoslovak Academy of Sciences, Praha, 1981. pp. 176--180.

Persistent URL: http://dml.cz/dmlcz/701249

\section{Terms of use:}

(C) Institute of Mathematics of the Academy of Sciences of the Czech Republic, 1981

Institute of Mathematics of the Academy of Sciences of the Czech Republic provides access to digitized documents strictly for personal use. Each copy of any part of this document must contain these Terms of use.

This paper has been digitized, optimized for electronic delivery
and stamped with digital signature within the project $D M L-C Z$ :
The Czech Digital Mathematics Library http://project.dml.cz


On infinite-dimensional marifolcs

H.Toruniczyk

Let I denote the segment $[-1,1], Q$ the Hilbert cube $I^{\infty}$ and $E_{2}$ the separable H1lbert space of square-summable sequences. In [4] and [5], the author has proved the following characterizations of spaces locally homeomorphic to $Q$ or $I_{2}$, respectively:

1. A locally compact $A N R-s p a c e, ~ X$, is a $Q$-manifold $1 f f$ the following condition is satisfled for each n:

$(\equiv)_{n}$ any map $1: I^{n} \times\{1,2\} \rightarrow X$ can arbitrarily closely be approximated by maps $B$ with $G\left(I^{n} \times 1\right) \cap B\left(I^{n} \times 2\right)=\varnothing$.

2. A separable complete-metrizable ANR-space, $X$, is an $I_{2}-\operatorname{manifold}$ iff

(zx) given an open covering $U$ of $x$ and a map $I: D \rightarrow X$, where $D$ is the disfoint union $I^{0} \cup I^{1} \cup \ldots$. there is a map $E: D \rightarrow X$ such that $\left\{g\left(I^{n}\right)\right\}$ n is a discrete collection in $X$ and $g$ is $U$-close to $f$

(1.e $\{f(d), g(a)\}$ refines $U, \forall a \in D$ ).

Here, we present an application of 2 to showing that certain topological groups are actually $I_{2}$-manifolds, and we report on some recent results of $\mathrm{B} . J . D a v e r m a n$ and J.Falsh related to result 1.

1. Topological Eroups which are Hilbert manifolds. T.Dobrowolski and the autor have jointly proved the following result:

Theorem ( [3]). Let $G$ be a metrizable topological group and I Its separable complete-metrizable subspace which is multiplicative (1.e. $1 \in X$ and $x \in X$ for $x, y \in X$ ). In order that $X$ be an $I_{2}$ manffold it suffices that $X \in A F E$ and no refghbourhood of 1 in $X$ 
be totelly baunded in the right structure of $G$.

Combined with earlier known facts this shows the following:

Corollary 1. Let $X$ be a complete-metrizable separable ANR. If $X$ adilts a topological group structure then either this is a Ile Eroup structure, and $X$ is a finite-dimensional manifold, or $X$ is an $I_{2}$-manifold.

Corollary 2. Let $X$ be a separable closed convex subset of a Banach space (or of a $B_{0}$-space). Then, $Z$ is either homeomorphic to $I_{2}$ or Is locally compact (and then homeomorphic to one of the sets $I^{k} \times R^{l} \times[0,1)^{m}$ where $k \leq \infty, I<\infty, m \leq 1$ and $m i n(m, I)=0$; see [1]).

Question: Do the analogues of the above corollarles hold true for non-separable spaces $X ?$ (C.I. the characterization of non-separable Hilbert manifolds in [5]).

Outline of the proof of the Theorem. Let a be a right-invariant metric for G. Me $11 x \quad U$ and $I=D \rightarrow X$ in (xz) and let

$$
\begin{aligned}
& \alpha(x)=\sup _{\left\{\text {Ist }_{d}(x, x \backslash U): v \in U\right\} / 2, x \in x,} \\
& D_{k}=\{d \in D: \alpha f(d) \geqslant 1 / k\}, k=1,2, \ldots
\end{aligned}
$$

Using the lact that no neighbourhood of 1 in $X$ is totally bounded in the metric d we construct sequences $\left\{B_{k}: D \rightarrow X\right\}_{k} \geqslant \theta$ and $\left\{\varepsilon_{k}\right\}_{k} \geqslant 0<(0 ; \infty)$ so that the following conditions hold for $k \geqslant 1$

(1) $B_{k}=I$ on $D \backslash D_{k+1}$ and $B_{k}=B_{k-1}$ on $D_{k-1}$;

$(2)_{k} \quad \mathrm{~d}\left(E_{k}\left(I^{\tilde{n}} \cap D_{k}\right), E_{k}\left(I^{\tilde{m}}\right)>\dot{\varepsilon}_{k}\right.$ for $m<n$;

$(3)_{k} d\left(\varepsilon_{k}, E_{k-1}\right)<\varepsilon_{k-1} / 4$ 
4) $k \varepsilon_{k}<\min \left\{1 / k, \varepsilon_{k-1} / 4\right\} \quad$ (Fere, $\varepsilon_{0}=1$ ).

It is not difficult to see that $\bar{B}=11 \mathrm{~m} \varepsilon_{k}$ is the map desired 1n (zx). (Hint: First check that $d(g(x) ; f(x))<\alpha f(x)$ for $x \in D$. To show that $\left\{g\left(I^{n}\right)\right\}_{n \geqslant 0}$ is discrete assume that $\left(g\left(x_{1}\right)\right)_{1}$ converges to a point $y \in X$ and distinct $x_{1}^{\prime} s$ belong to distinct cells in $D$. Then inf $\alpha f\left(x_{1}\right)>0$ - for otherwise $\left(f\left(x_{1}\right)\right)$ would contain a sub-sequence converging to $\mathrm{g}$, ylelaing $\alpha(y)=0$. Thus there is a $k \in \mathbb{N}$ with $\left\{x_{1}\right\}_{1=0}^{\infty}<D_{k}$ and (1) and $(2)_{k}$ yield $a\left(g\left(x_{1}\right), g\left(x_{j}\right)\right) \geqslant \varepsilon_{k}$ for $1 \neq j$. This contradicts the assumed convergence of $\left(g\left(x_{1}\right)\right)$.

The construction of the $\delta_{k}^{j} s$ and $\varepsilon_{k}{ }^{\prime}$ (outline). Assume for simplicity that $\dot{D}_{1}=D$. In this case the sequences will terminate after the first step, which is as follows, Let $\varepsilon_{1}$ be so small that no compact set in $G 18$ an $\varepsilon_{1}$ - net in

$B=\{x \in X: d(x, 1)<1\}$. We let $B_{1}\left|I^{0}=I\right| I^{0}$ and, if $B_{1} \mid I^{0} \cup \ldots \cup I^{n} \quad$ Is already defined, we select $p \in B$ with

$$
a(p, x)>\varepsilon_{1} \text { for } x \in\left\{a b^{-1}: a, b, \in g_{1}\left(I^{0} \cup \ldots \cup I^{n}\right) \cup f\left(I^{n+1}\right)\right\}
$$

and we put $g_{1}(x)=p f(x)$ for $x \in I^{n+1}$. In this way we inductively define $g_{1} \mid I^{n}$. so that the resulting map $B_{1}$ satisfies $(2)_{1}$. The general case (where no $D_{1}$ equals $D$ ) is technically more Involred but follows the same 1dea. See [3] for detalls.

82. Homology characterizations of 2-manifolds. B.J.Daverman and J. Falsh have recently showed that, in the result 1, the "disjoint n-cube property" of $(x)_{n}$ can for $n>2$ be replaced by a cisfointress property in homologles. To be wore specific let us 
say that, whenever $(U, V)$ is an open pair in $X$ and $\alpha \in H_{z}(U, \nabla)$ (Integer coefficients), a compact pair $(A, B) \dot{C}(U, V)$ is said to be a Cech carler for $\alpha$ iff any nelghbourhood of $(A, B)$ in $(U, V)$ contains a cycle homologous to $\alpha$ in $(U, V)$.

Theorem ( [2]). Let $X$ be a locally compact AlR. Then, $X$ is a Q-manifold iff it satisfies $(x)_{2}$ and the folloring condition: (x)' given open pairs $\left(U_{1}, V_{1}\right)$ in $X$ and relative cycles $\alpha_{1} \in H_{z}\left(U_{1}, V_{1}\right), 1=1,2$, there are Cech carriers $\left(A_{1}, B_{1}\right)$ for $\alpha_{1}$ with $A_{1} \cap A_{2}=\varnothing$.

It is unknown whether (x)' is satisfied by any infinite-dimensional homology manifold $X$ (1.e., by any locally compact All such that $H_{z}(X, X \backslash\{x\})=0$ for each $\left.x \in X\right)$. It is easy to show that $(x)$ ' Is satisfied if the homology manifold has the property that any relative cycle in it admits a Iinite-dimensional Cech carrier;see [2]. Also, property (x)' is relatively easy to prove for certain CE-1mages of Q-manifolds, and thereby can be used to prove that these Images are manifolds themselfes. A sample application is:

Corollary ( [2]). If $X$ is a space such that $X \times I^{n} \cong 0$ for some finite $n$ then $X \times I^{2} \cong Q$.

It is unknown whether, in the statement above, $I^{2}$ can be replaced by $I$. This is related to the open problem whether $X \times I$ satisfies $(x)_{2}$ for any infinite-dimensional homology manifold; this problem is of great interest also formanifolds of inte dimension $n \geqslant 4$. The author has recently abserved that, at least, $X \times D$ satisfies $(x)_{2}$ for any $X$ as above and $D$ a dendron with $a$ dense set of separating points. Denoting by $p: D \rightarrow I$ the natural retraction having sto ros-triviel polnt laverses, all of rhich are 
dendra, one thus faces the following

2uestion: If $X \times D \cong Q$, is ${ }^{1} X \times P: X \times D \rightarrow Z \times I$ approximable by homeomorphisms?

\section{References}

[1] C.Sessaga and A.Pelczynski, Selected Topics in Infinite-Dimensional Topology, Narszawa 1975.

[2] B.J.Daverman and J.Walsh, Cech homology characterization of infinite-dimensional manifolds, preprint.

[3] T.Dobrowolski and H.Torunczyk, Complete separable AIR's admitting a group structure are Hilbert manifolds, to appear on Topologs and 1ts Appl.

[4] Hotorunczyk, On CB - images of the H1lbert cube and characterization of Q-manifolds, to appear in Fund.Hath.

[5] H.Torunczyk, Characterizing Hilbert space topology, to appear in Fund.liath.

Editorlal note:

This is an abstract of a talk presented by the author at the 8th Winter School (1980) 